\title{
Sleep-Disordered Breathing in Pregnancy
}

\author{
Louise M. O'Brien ${ }^{1}$
}

Published online: 20 October 2016

(C) Springer International Publishing AG 2016

\begin{abstract}
Sleep-disordered breathing (SDB) during pregnancy has emerged as a predictor of both maternal and fetal adverse outcomes. Data suggest that SDB during pregnancy is associated with maternal gestational hypertension and preeclampsia as well as gestational diabetes. Furthermore, there are increasing reports that SDB may play a role in fetal growth restriction, preterm birth, increased cesarean sections, and increased neonatal intensive care unit (NICU) admissions. The public health impact of unrecognized maternal SDB is therefore immense. Treatment of SDB in the general population has been shown to improve cardiovascular outcomes and daytime functioning, and although no large intervention trial has been conducted in pregnant women, small pilot studies demonstrate promising results.
\end{abstract}

Keywords Sleep-disordered breathing · Pregnancy · Hypertension $\cdot$ Preeclampsia $\cdot$ Gestational diabetes $\cdot$ Fetal growth restriction $\cdot$ Small-for-gestational-age $\cdot$ Preterm birth . Cesarean section $\cdot$ Neonatal intensive care unit $\cdot$ Positive airway pressure

\section{Introduction}

Sleep-disordered breathing (SDB) is a common condition characterized by nocturnal airway collapse and which affects

This article is part of the Topical Collection on Women and Sleep

Louise M. O'Brien

louiseo@med.umich.edu

1 Sleep Disorders Center, Department of Neurology, Department of Obstetrics \& Gynecology, University of Michigan, Med Inn Building Rm C736, Ann Arbor, MI 48109-5845, USA up to $35 \%$ of pregnant women in the general population and up to $85 \%$ of those with hypertensive pregnancies by the third trimester [1-3]. It is strongly associated with obesity; compared with lean women $\left(<25 \mathrm{~kg} / \mathrm{m}^{2}\right)$, overweight women $\left(25-29.9 \mathrm{~kg} / \mathrm{m}^{2}\right)$ are reported to have a 3.69 -fold higher odds of obstructive sleep apnea (OSA) risk (95\%CI 1.82-7.50) [4]. The corresponding adjusted odds ratio (OR) for obese women $\left(\geq 30 \mathrm{~kg} / \mathrm{m}^{2}\right.$ ) was 13.23 (95\%CI 6.25-28.01) [4]. Furthermore, weight gain of only $10 \%$ has been associated with a 6 -fold increase in the development of significant SDB [5]. Strikingly, data suggest that more than two thirds of reproductive age women are overweight or obese, and almost half of pregnant women gain excessive weight $[6,7]$. As discussed below, SDB has been associated with poor maternal and fetal outcomes, yet treatment trials are remarkably scarce. Given the potential implications for the health of both mother and child, evaluation and management of pregnant women with SDB is a clinical imperative.

\section{SDB and Maternal Outcomes}

The cardiovascular effect of untreated SDB has been welldocumented in the non-pregnant population, with strong evidence for a causal relationship between SDB and hypertension and metabolic dysfunction [8,9]. Similar associations have been reported in studies of pregnant women, as described below:

\section{Gestational Hypertension/Preeclampsia}

Hypertensive disorders of pregnancy affect up to $10 \%$ of pregnancies [10] and contribute to serious maternal and fetal morbidities in the short and long term. Of particular concern is preeclampsia, characterized by new-onset hypertension and 
proteinuria after a 20 -week gestation. The incidence of hypertensive disorders of pregnancy has increased two-fold in recent decades [11].

Several large (>1000 women) studies of self-report habitual snoring demonstrate an approximately 2 -fold increase in gestational hypertension/preeclampsia $[1,2,12,13]$. In a predominantly Hispanic population, Antony et al. [13] did not find that snorers had an increase in gestational hypertension but did find an increased relative risk (RR) of 2.45 (95\%CI 1.543.24) for preeclampsia after adjustment for other variables. Similarly, Higgins et al. [14] found that $10.1 \%$ of women who screened positive on the Berlin questionnaire [15], a commonly used screening tool for risk of OSA, had preeclampsia compared to only $1.8 \%$ of those who screened negative $(p<0.01)$. In a large cohort study, O'Brien et al. has demonstrated that women who develop pregnancy-onset snoring - but not women with chronic snoring - have an OR of 2.36 (95\% CI 1.45-3.77) for gestational hypertension and an OR of 1.59 (95\%CI 1.06-2.37) for preeclampsia after adjustment for covariates, particularly body mass index (BMI) [16]. These findings raise the possibility that the new-onset snoring during gestation, perhaps with little time for physiological adaptation to occur, may adversely impact maternal blood pressure.

Other, smaller studies of snoring and hypertension have also demonstrated such a relationship [17-19]. In a study of $n=209$ women, compared to non-snorers, women who reported snoring in the first trimester had an OR of 4.0 (95\% CI 1.3-11.9) for developing gestational hypertension [18]. However, women who were classified as high risk (using the modified Berlin questionnaire) during all three trimesters had 30 times increased odds (95\%CI 3.6-253.0) of developing gestational hypertension. Witnessed apnea, another key symptom, has been reported to be associated with an increased risk for gestational hypertension and preeclampsia, with one study suggesting that the risk is increased to 8-fold [17].

As well as many symptom-based studies, several studies of objectively measured SDB support an association with hypertension [20•, 21-23], although some studies do not [24, 25]. O'Brien et al. has demonstrated that approximately half of all women with preeclampsia and one quarter of women with gestational hypertension, have unrecognized obstructive sleep apnea (OSA), compared to one in five normotensive women [20 ]. Importantly, approximately $10 \%$ of hypertensive women have severe obstructive sleep apnea. Notably, hypertensive women who reported habitual snoring were significantly more likely than non-snoring hypertensive women to have undiagnosed OSA (53 vs. $24 \%, p=0.03$ ), suggesting that snoring in the setting of hypertension warrants closer scrutiny [20•].

After stratification by obesity, we still found that the pooled RR for OSA in snoring women with hypertension compared to non-snoring hypertension was 2.0 (95\%CI 1.4-2.8) [20•]. In a study of obese women, OSA was associated with an OR of $3.55(1.12-11.3)$ for preeclampsia after controlling for maternal age, chronic hypertension, prior preeclampsia, BMI, and pre-gestational diabetes [23]. However another study by the same group did not find an increase in preeclampsia above the obesity-related increased risk [26]. Obesity is a significant risk factor for preeclampsia and for SDB; it is therefore challenging to determine the independent impact of SDB on hypertensive status. Moreover, there is near universal adjustment for obesity in most studies, rather than investigation of the true role of obesity or gestational weight gain in the pathway of SDB and hypertension, and this may produce biased estimates. For example, it is currently unknown whether maternal weight confounds or mediates the relationship between SDB and hypertension.

Large epidemiological studies that have included women with objectively diagnosed OSA found that those with OSA had an increased risk of pregnancy-induced hypertension (adjusted RR 1.43; 95\%CI 1.18-1.73) [27] and preeclampsia (adjusted ORs between 1.6-2.5) [28, 29•] compared to women without OSA. In addition to preeclampsia, using a national sample of inpatient data, OSA is associated with an increased risk of severe maternal morbidity including cardiomyopathy (OR 9.0, 95\%CI 7.5-10.9), pulmonary embolism (OR 4.5, 95\%CI 2.3-8.9), eclampsia (OR 5.4, 95\%CI 3.3-8.9), and hospital death (OR 5.3, 95\%CI 2.4-11.5) [29•]. While clearly important findings, large studies rely on diagnostic coding are therefore likely to have significant misclassification since $>90 \%$ of women with SDB are undiagnosed [30]. Furthermore, treatment effects cannot be accounted, further adding to misclassification. The finding of increased hospital deaths is of concern, particularly since the maternal mortality ratio has doubled in the United States between 1990 and 2003 and half of such deaths are preventable [31].

With the increasing number of studies now available, systematic reviews and meta-analyses have been published which report a two-fold increase in gestational hypertension and preeclampsia among women with SDB [32,33], although this effect may only be higher in the subgroup of women with a BMI $>30 \mathrm{~kg} / \mathrm{m}^{2}($ RR 1.94, 95\%CI 1.15-3.26; $\mathrm{p}=0.013)$ and not in normal weight women (RR 1.69; $95 \%$ CI 0.62 to 4.61; $\mathrm{p}=0.304)$ [34]. Until the complex relationship between SDB, obesity, and hypertension has been delineated, however, such effects remain speculative.

\section{Gestational Diabetes}

Gestational diabetes mellitus (GDM) affects approximately $16 \%$ of pregnant women. Although it is strongly related to pre-pregnancy obesity [35], pregnancy itself represents a state of insulin resistance and GDM can result when insulin secretion fails to counterbalance the natural increased insulin resistance. It is associated with significant morbidities of both the mother and the offspring, including, preeclampsia, 
macrosomia, cesarean section, later life maternal diabetes, childhood obesity and both maternal and childhood cardiovascular disease.

Symptom-based studies have generally found that GDM is associated with SDB [2, 36-39]. In a prospective cohort of $\mathrm{n}=189$ women, the odds of GDM in frequent snorers was reported to be 6.7 (95\%CI 1.4-33.8) [36]. Similarly, in a study of $n=1290$ women recruited in early pregnancy, snoring was associated with a 1.86-fold increased risk of GDM (RR 1.86, 95\%CI 0.88-3.94) [37]. The risk of GDM was particularly elevated among overweight women who snored. Compared with lean women who did not snore, those who were overweight and snored had a 6.9-fold increased risk of GDM (95\% CI 2.87-16.6) [37]. We have previously shown that although women with habitual snoring have higher blood glucose levels at the 1-h oral glucose tolerance test (124 vs. $117 \mathrm{mg} / \mathrm{dl}, \mathrm{p}<0.001$ ) and are more likely to have abnormal glucose levels ( $\geq 140 \mathrm{mg} / \mathrm{dl} ; 30 \%$ vs. $22 \% \mathrm{p}-0.003$ ) compared to non-snoring women, stratification by obesity demonstrated that only obese women (regardless of snoring status) had an increase in GDM [1]. Other studies have also failed to support an association between SDB and GDM [18, 23].

Several population-based studies of women with a diagnosis of OSA have also found mixed results. A retrospective cross-sectional national inpatient discharge database found that OSA was associated with an increased likelihood of GDM (adjusted OR 1.9, 95\% CI, 1.7-2.1) [29•]. A crosssectional population database using a diagnosis of OSA within 1 year prior to delivery reported that the odds of GDM increased almost two-fold (OR 1.6, 95\%CI 1.07-2.8) among 791 pregnant women diagnosed with OSA [28] although this study did not fully control the effect of obesity. However, more recently, a large population database from Australia has failed to find any association between a diagnosis of OSA and GDM [27].

Systematic reviews and meta-analyses have reported that SDB is associated with a two- to three-fold increased pooled odds of GDM [32, 33, 40]. Similar to hypertension discussed above, there is near universal adjustment or stratification of obesity rather than investigation of its true role in the pathway of SDB and GDM.

\section{SDB and Delivery Outcomes}

In addition to associations with maternal health, an initial report describing an association between maternal apnea and fetal heart rate decelerations was reported in the 1970's [41], but it was not until recent years that studies of SDB have begun to focus on the fetus. In 2000, Franklin et al. [42] found that infants born to snoring women were more likely to be growth restricted. Since then there have been a number of studies, although with conflicting findings. Relatively large cohort studies (>1000 women) utilizing habitual snoring as a marker of SDB have found higher risk of fetal growth restriction (RR 2.0, 95\%CI 1.0-3.8) [43] or small-for-gestationalage (SGA; OR 1.65, 95\%CI 1.02-2.66) [16]. However, other similarly large cohort studies have failed to support a finding of SGA in snoring women [44] or in those with a positive Berlin questionnaire [13].

Conversely, in some studies, a positive Berlin questionnaire has been associated with a larger median birth weight [14] and large-for-gestational-age (LGA) [13] although it is possible that the latter findings are related to maternal obesity since Olivarez et al. [45] also found higher birth weights in infants of mothers with a positive Berlin score but after stratifying by obesity the findings were no longer significant in women with a $\mathrm{BMI}<30 \mathrm{~kg} / \mathrm{m}^{2}$. These conflicting findings may be related to the wide spectrum of respiratory disturbance that is included in a query about SDB symptoms at only one time point. For example, O'Brien et al. has demonstrated that women who report chronic habitual snoring (i.e., snoring that was present prior to pregnancy) are more likely to have a newborn that is SGA, and that women who develop pregnancy-onset snoring do not demonstrate such a relationship [16]. Nonetheless, in the same cohort of women, selfreport of witnessed apnea was associated with LGA infants. However, in a regression model it was maternal diabetes and not witnessed apnea that was independently associated with LGA. The relationships between SDB, cardiovascular and metabolic milleu, maternal morbidity, and fetal growth are complex and cannot be fully delineated without large, longitudinal studies explicitly designed for this purpose.

Studies of fetal outcomes utilizing objective measurements of maternal sleep are limited. Most studies that have longitudinally recruited women fail to find a relationship between maternal OSA and birth weight [46, 47], SGA [46-49], or LGA [48]. Different polysomnographic systems were used across these studies and definitions of OSA varied by study. One large cross-sectional study that used data from a population-based database of $>4000$ women, found that OSA was associated with an increased risk of low birth weight and SGA [28]. Maternal obesity likely plays an important role as birth weight has been found to be lower in women with OSA when compared to infants of obese women but higher when compared to infants of non-obese controls [26]. However, data from a US national inpatient discharge database of almost 56 million women did not find any relationship with SGA [29•] and an Australian study of discharge records found that OSA was associated with LGA (OR 1.27, 95\%CI 1.04-1.55) [27]. While the latter very large population based studies provide important associations, interpretation must be made with caution since data were not captured regarding OSA treatment among women with a diagnosis, nor did the non-OSA comparison group undergo polysomnography to rule out OSA. Given that over $90 \%$ of women with OSA do 
not know that they have it [30], misclassification in these epidemiological studies is highly probable. Interestingly, systematic reviews/meta-analyses have also failed to confirm a difference in mean birth weight [32, 33], or SGA [34] but have found a higher incidence of low birth weight [32].

Importantly, fetal size at birth is a rather crude measure; trajectory of fetal growth is more informative. Fung et al. [46] failed to find a difference in birth weight or SGA in their small cohort. However, when plotting fetal size at 32 weeks' gestation compared to birth centile, they did find that fetal growth trajectory fell across centiles in late pregnancy in women who had OSA. Using serial fetal growth measures, Kneitel et al. has subsequently confirmed a slowing of fetal growth in the third trimester (Knietel under review) even when the infant would not be considered SGA at birth.

\section{Preterm Birth}

Few studies of pregnancy outcomes and SDB symptoms have investigated preterm birth (PTB), usually due to study designs recruiting women in late pregnancy. Of those that have, most do not find any relationship either in cohort [43, $50,51]$ or cross-sectional studies [2, 19]. A positive Berlin score has been associated with PTB in a large cohort study in an unadjusted model, but after adjustment for confounders including smoking, parity, BMI, hypertension, and GDM the relationship was no longer significant [13]. Recently, a prospective study of $n=1345$ Thai women found that with stratified analyses, after adjusting for confounding factors, a high risk of OSA was associated with an increased risk of spontaneous preterm delivery, OR 2.45, (95\% CI 1.20-5.02), but not with preterm premature rupture of membranes or medically indicated preterm delivery [52]. One cross-sectional study found that report of gasping for air demonstrated an increased odds of PTB after accounting for maternal age, smoking, and multifetal pregnancies with an OR of 1.9 (95\%CI 1.1-3.5) but no data were provided after adjustment for other factors known to be related to PTB such as preeclampsia [2].

Similar to many symptom-based studies, several studies using objective measures of OSA have failed to find evidence of a link with PTB [23, 48, 49] although one study did find an increase in PTB $(<37$ weeks gestation and $<32$ weeks gestation) in women with OSA in a retrospective cohort [26]. Mean gestational age in women with OSA may be slightly shorter than controls ${ }^{44}$ although a recent study has not been able to support these findings [47]. Nonetheless, data from population-based datasets demonstrates an increase in PTB [28] or "early onset delivery" [29•], and similarly, one systematic review and one metaanalysis of cohort studies has reported a two-fold increase in PTB with maternal SDB $[32,34]$.

\section{Cesarean Section}

Sleep deprivation and fatigue have previously been reported to increase the incidence of cesarean section [28, 53-55]. It is only in recent years that studies of SDB have reported mode of delivery. Subjective measures of SDB have shown an association between snoring and cesarean delivery [2, 13, $16,18,50]$, particularly those that are unplanned $[2,13,16]$ A newly published longitudinal study that queried women at each trimester has reported that, compared to women who do not snore, those who snore during one, two, or all three trimesters have a significantly increased odds for cesarean section; OR 5.3 (95\%CI 1.7-16.3), 4.9 (95\%CI 1.8-13.1), and 5.1 (95\%CI 1.9-14.9) respectively, although it is not clear whether this was adjusted for other covariates [18]. Accounting for known risk factors for cesarean delivery as well as the chronicity of snoring, we have shown that both chronic and pregnancy-onset snoring are independently associated with elective cesarean delivery (OR 2.25, 95\% CI $1.22-4.18$ and OR $1.70,95 \%$ CI $1.13-2.57$ respectively) [16]. Furthermore, only pregnancy-onset snoring remained independently associated with emergency cesarean delivery; OR 1.68 (95\%CI 1.22-2.30). However, in a study that stratified data by obesity, the relationship between snoring and cesarean delivery was lost [50].

An objective diagnosis of OSA is also associated with cesarean delivery $[23,26,28,29 \bullet]$. Women with OSA are more likely to have a cesarean delivery for arrest of labor compared to both normal weight women and obese women (58\% vs. $17 \%, \mathrm{p}<0.001$ and $58 \%$ vs. $46 \%, \mathrm{p}=0.04$ respectively) [26]. Large population datasets in the US [29•] and Taiwan [28] report an increased odds ratio for cesarean section, OR 1.12 (95\%CI 1.01-1.23) and OR 1.74 (95\%CI 1.48-2.04) respectively, although a recent population based study from Australia did not find a greater likelihood of cesarean section [27]. As with all secondary analyses of large datasets, these studies have high risk of misclassification for the key exposure variable (diagnosis of OSA) and cannot fully control for key variables such as obesity which are identified via ICD coding. A meta-analysis supports the notion of increased cesarean deliveries [34], Moreover, it is plausible that maternal SDB could be associated with operative delivery via associated fatigue that could result in exhaustion and ultimately prolongation of labor and cesarean section $[16,56]$.

\section{Neonatal Intensive Care Unit Admission}

Reasons for neonatal intensive care unit (NICU) admission are varied, and the proportion of infants admitted for any given indication can be small. Few studies have investigated NICU admission in the setting of maternal SDB, and of those that have, findings are mixed. A study of $n=276$ Korean women did not demonstrate a relationship 
with a positive Berlin screen and NICU admission [50], while a US study of $n=1153$ women using the same tool found that the frequency of level 2/3 NICU admission was higher in women with a positive screen ( 23 vs. $16 \%$, $p=0.002$ ) [13]. These data are very similar to findings from a Ghanaian population where NICU admission was higher in snoring women compared to non-snorers (23 vs. $15 \%)$ [19], although in the latter case, it did not reach statistical significance due to a small total sample $(n=220)$. In a large cohort, we have found a trend for newborn transfer to the treatment room, triage, or the NICU with the highest frequency of transfer in infants born to women with chronic, as opposed to pregnancyonset SDB [16]. Although a greater proportion of infants in the chronic snoring group were admitted to the NICU, this did not reach statistical significance.

Infants of women with objectively diagnosed OSA have been reported to have increased NICU admission compared to those without OSA (26 vs. $12 \%, p=0.01$ ) [26] with the OR for NICU admission in women with OSA reported as being more than 3-fold those without (OR 3.39, 95\%CI 1.23-9.32) [23]. A systematic review and meta-analysis, each of four studies (three overlapping), revealed that SDB was associated with a two-fold increase in NICU admission [32, 34]. However, in a population based data analysis of $>636,000$ hospital stays, Bin et al. [27] observed an association between OSA and admission to the NICU/special care nursery (SCN) for term but not preterm babies. These authors postulated that preterm infants might be admitted to the NICU/SCN on the basis of prematurity alone, while maternal OSA appears to increase the likelihood of NICU/SCN admission for term infants.

\section{Mechanisms}

The postulated mechanisms of sleep disruption that affect cardiovascular morbidity in non-pregnant individuals are remarkably similar to the biological pathways for preeclampsia. Although the pathogenic process for preeclampsia most likely originates in the placenta early in pregnancy, with abnormal implantation and vascular modeling, both the pathways for preeclampsia and for the cardiovascular impact of SDB include hypoxia, endothelial dysfunction, oxidative stress, and inflammation [57]. These shared mechanistic pathways have been discussed previously [58]. It is plausible that the normal maternal inflammatory response to pregnancy could be exacerbated by SDB, predisposing pregnant women to preeclampsia [58, 59]. Indeed, a hypothesis paper has postulated that disturbed sleep in early pregnancy contributes to an increased inflammatory response that may disrupt the normal vascular modeling of blood vessels [60]. Interestingly, the placentas of women with SDB have evidence of fetoplacental hypoxia [61] and increased angiogenesis [62].

\section{Treatment of SDB in Pregnancy}

The standard treatment for SDB is positive airway pressure (PAP). Despite PAP being safe and effective, treatment studies are lacking in pregnant women. However, available data from small studies show that use of PAP in hypertensive pregnancies or those at risk for preeclampsia is associated with improved blood pressure $[63,64]$ but may not be sufficient to prevent negative pregnancy outcomes [65].

During an apneic episode, there are surges in sympathetic output followed by an abrupt cessation at the termination of the apnea [66]; marked augmentation of this hemodynamic response to obstructive apneas is observed in preeclampsia [67]. Notably, the adverse hemodynamic changes associated with sleep in women with preeclampsia can be minimized by PAP therapy [63, 68]. Although Edwards et al. [63] found a marked decrease in blood pressure with only one night of PAP treatment, others have failed to replicate this finding with a single night of therapy [69]. Indeed, a recent case study suggests that more than one night is required to demonstrate improvement: PAP therapy was initiated in a woman at 29 weeks gestation with early onset preeclampsia and severe OSA [70]. Her clinical, biochemical, and angiogenic markers of preeclampsia improved over the following weeks, after which they deteriorated until she was delivered at 35 weeks. Despite the preterm delivery, PAP use allowed a prolongation of the pregnancy by 30 days, which vastly improved neonatal outcome without jeopardizing maternal health.

Recent data, again from women with preeclampsia, suggest that PAP use is also associated with improvements in fetal movements [71], a known sign of fetal well-being. Reduced fetal movements were found in women with preeclampsia, many of who had SDB, and improvement in fetal movements were observed following PAP use. Unfortunately, since all women had preeclampsia and most had airflow limitation, it was not possible to determine whether the increased fetal movements associated with PAP use occurred via treatment of underlying SDB or due to an effect of PAP on the cardiovascular system independent of the presence of SDB. In a secondary analysis of fetal growth trajectory, we have recently demonstrated that OSA during pregnancy is associated with a slowing of fetal growth but that fetal growth is similar to non-OSA controls in women who were compliant with PAP therapy (Knietel under review). Collectively, the limited data on PAP use in pregnancy is suggestive in that it may improve maternal cardiovascular control and possibly fetal wellbeing. Intervention studies in women with SDB are therefore urgently needed. 


\section{Conclusion}

Based on current evidence, untreated SDB is an independent risk factor for hypertensive disorders of pregnancy, gestational diabetes, and possibly untoward fetal outcomes. Since many studies are based on either symptoms of SDB or large population-based datasets, large longitudinal objective trials, such as a recently completed study in the USA [72•], will be critical to further investigate these associations and address major confounders such as obesity and gestational weight gain. Given the possibility that PAP therapy could improve both maternal and fetal health, large treatment intervention utilizing PAP are warranted. The high prevalence of SDB during pregnancy suggests that targeting maternal SDB will allow novel therapeutic interventions that will reduce the burden of poor pregnancy outcomes and will ultimately have a positive long-term impact on both the mother and the offspring.

\section{Compliance with Ethical Standards}

Conflict of Interest Louise M. O'Brien has received grants from the American Sleep Medicine Foundation and autoPAP devices for research purposes from Phillips Respironics Inc.

Human and Animal Rights and Informed Consent The studies that are cited all obtained consent from participants.

\section{References}

Papers of particular interest, published recently, have been highlighted as:

- Of importance

1. O'Brien LM, Bullough AS, Owusu JT, et al. Pregnancy-onset habitual snoring, gestational hypertension, and preeclampsia: prospective cohort study. Am J Obstet Gynecol. 2012;207(6):487. e1-9.

2. Bourjeily G, Raker CA, Chalhoub M, et al. Pregnancy and fetal outcomes of symptoms of sleep-disordered breathing. Eur Respir J. 2010;36(4):849-55.

3. Izci B, Martin SE, Dundas KC, et al. Sleep complaints: snoring and daytime sleepiness in pregnant and pre-eclamptic women. Sleep Med. 2005;6(2):163-9.

4. Rice JR, Larrabure-Torrealva GT, Luque Fernandez MA, et al. High risk for obstructive sleep apnea and other sleep disorders among overweight and obese pregnant women. BMC Pregnancy Childbirth. 2015;15:198.

5. Peppard PE, Young T, Palta M, et al. Longitudinal study of moderate weight change and sleep-disordered breathing. JAMA. 2000;284(23):3015-21.

6. Chu SY, Callaghan WM, Bish CL, et al. Gestational weight gain by body mass index among US women delivering live births, 2004 2005: fueling future obesity. Am J Obstet Gynecol. 2009;200(3): 271. e1-7.
7. Hillemeier MM, Weisman CS, Chuang C, et al. Transition to overweight or obesity among women of reproductive age. J Womens Health (Larchmt). 2011;20(5):703-10.

8. Nieto FJ, Young TB, Lind BK, et al. Association of sleepdisordered breathing, sleep apnea, and hypertension in a large community-based study. Sleep Heart Health Study. JAMA. 2000;283(14):1829-36.

9. Peppard PE, Young T, Palta M, et al. Prospective study of the association between sleep-disordered breathing and hypertension. N Engl J Med. 2000;342(19):1378-84.

10. Duley L. The global impact of pre-eclampsia and eclampsia. Semin Perinatol. 2009;33(3):130-7.

11. Wallis AB, Saftlas AF, Hsia J, et al. Secular trends in the rates of preeclampsia, eclampsia, and gestational hypertension, United States, 1987-2004. Am J Hypertens. 2008;21(5):521-6.

12. Ursavas A, Karadag M, Nalci N, et al. Self-reported snoring, maternal obesity and neck circumference as risk factors for pregnancyinduced hypertension and preeclampsia. Respiration. 2008;76(1): 33-9.

13. Antony KM, Agrawal A, Arndt ME, et al. Association of adverse perinatal outcomes with screening measures of obstructive sleep apnea. J Perinatol. 2014;34(6):441-8.

14. Higgins N, Leong E, Park CS, et al. The Berlin Questionnaire for assessment of sleep disordered breathing risk in parturients and non-pregnant women. Int J Obstet Anesth. 2011;20(1):22-5.

15. Netzer NC, Stoohs RA, Netzer CM, Clark K, Strohl KP. Using the Berlin Questionnaire to identify patients at risk for the sleep apnea syndrome. Ann Intern Med. 1999;131(7):485-91.

16. O'Brien LM, Bullough AS, Owusu JT, et al. Snoring during pregnancy and delivery outcomes: a cohort study. Sleep. 2013;36(11): 1625-32.

17. Perez-Chada D, Videla AJ, O'Flaherty ME, et al. Snoring, witnessed sleep apnoeas and pregnancy-induced hypertension. Acta Obstet Gynecol Scand. 2007;86(7):788-92.

18. Sharma SK, Nehra A, Sinha S, et al. Sleep disorders in pregnancy and their association with pregnancy outcomes: a prospective observational study. Sleep Breath. 2016;20(1):87-93.

19. Owusu JT, Anderson FJ, Coleman J, et al. Association of maternal sleep practices with pre-eclampsia, low birth weight, and stillbirth among Ghanaian women. Int J Gynaecol Obstet. 2013;121(3):261-5.

20. O'Brien LM, Bullough AS, Chames MC, et al. Hypertension, snoring, and obstructive sleep apnoea during pregnancy: a cohort study. BJOG. 2014;121(13):1685-93. This study demonstrates that snoring in the setting of a hypertensive pregnancy warrants clinical evaluation

21. Reid J, Skomro R, Cotton D, et al. Pregnant women with gestational hypertension may have a high frequency of sleep disordered breathing. Sleep. 2011;34(8):1033-8.

22. Facco FL, Lappen J, Lim C, et al. Preeclampsia and sleepdisordered breathing: a case-control study. Pregnancy Hypertens. 2013;3(2):133-9.

23. Louis J, Auckley D, Miladinovic B, et al. Perinatal outcomes associated with obstructive sleep apnea in obese pregnant women. Obstet Gynecol. 2012;120(5):1085-92.

24. Olivarez SA, Maheshwari B, McCarthy M, et al. Prospective trial on obstructive sleep apnea in pregnancy and fetal heart rate monitoring. Am J Obstet Gynecol. 2010;202(6):552. e1-7.

25. Facco FL, Liu CS, Cabello AA, et al. Sleep-disordered breathing: a risk factor for adverse pregnancy outcomes? Am J Perinatol. 2012;29(4):277-82.

26. Louis JM, Auckley D, Sokol RJ, et al. Maternal and neonatal morbidities associated with obstructive sleep apnea complicating pregnancy. Am J Obstet Gynecol. 2010;202(3):261. e1-5.

27. Bin YS, Cistulli PA, Ford JB. Population-based study of sleep apnea in pregnancy and maternal and infant outcomes. J Clin Sleep Med. 2016;12(6):871-7. 
28. Chen YH, Kang JH, Lin CC, et al. Obstructive sleep apnea and the risk of adverse pregnancy outcomes. Am J Obstet Gynecol. 2012;206(2):136. e1-5.

29. Louis JM, Mogos MF, Salemi JL, et al. Obstructive sleep apnea and severe maternal-infant morbidity/mortality in the United States, 1998-2009. Sleep. 2014;37(5):843-9. This hospital discharge data showed serious maternal morbidities in women with a diagnosis of OSA.

30. Young T, Evans L, Finn L, et al. Estimation of the clinically diagnosed proportion of sleep apnea syndrome in middle-aged men and women. Sleep. 1997;20(9):705-6.

31. Trends in maternal mortality: 1990 to 2013. Estimates by WHO, UNICEF, UNFPA, The World Bank and the United Nations Population Division. Geneva: World Health Organization; 2014. Available from: http://www.who. int/reproductivehealth/publications/monitoring/maternalmortality-2013/en/

32. Ding XX, Wu YL, Xu SJ, et al. A systematic review and quantitative assessment of sleep-disordered breathing during pregnancy and perinatal outcomes. Sleep Breath. 2014;18(4):703-13.

33. Pamidi S, Pinto LM, Marc I, et al. Maternal sleep-disordered breathing and adverse pregnancy outcomes: a systematic review and metaanalysis. Am J Obstet Gynecol. 2014;210(1):52. e1-52 e14.

34. Xu T, Feng Y, Peng H, et al. Obstructive sleep apnea and the risk of perinatal outcomes: a meta-analysis of cohort studies. Sci Rep. 2014;4:6982.

35. Coustan DR, Lowe LP, Metzger BE, et al. The Hyperglycemia and Adverse Pregnancy Outcome (HAPO) study: paving the way for new diagnostic criteria for gestational diabetes mellitus. Am J Obstet Gynecol. 2010;202(6):654. e1-6.

36. Facco FL, Grobman WA, Kramer J, et al. Self-reported short sleep duration and frequent snoring in pregnancy: impact on glucose metabolism. Am J Obstet Gynecol. 2010;203(2):142. e1-5.

37. Qiu C, Enquobahrie D, Frederick IO, et al. Glucose intolerance and gestational diabetes risk in relation to sleep duration and snoring during pregnancy: a pilot study. BMC Womens Health. 2010;10:17.

38. Reutrakul S, Zaidi N, Wroblewski K, et al. Sleep disturbances and their relationship to glucose tolerance in pregnancy. Diabetes Care. 2011;34(11):2454-7.

39. Ugur MG, Boynukalin K, Atak Z, et al. Sleep disturbances in pregnant patients and the relation to obstetric outcome. Clin Exp Obstet Gynecol. 2012;39(2):214-7.

40. Luque-Fernandez MA, Bain PA, Gelaye B, et al. Sleep-disordered breathing and gestational diabetes mellitus: a meta-analysis of 9 , 795 participants enrolled in epidemiological observational studies. Diabetes Care. 2013;36(10):3353-60.

41. Joel-Cohen SJ, Schoenfeld A. Fetal response to periodic sleep apnea: a new syndrome in obstetrics. Eur J Obstet Gynecol Reprod Biol. 1978;8(2):77-81.

42. Franklin KA, Holmgren PA, Jonsson F, et al. Snoring, pregnancyinduced hypertension, and growth retardation of the fetus. Chest. 2000;117(1):137-41.

43. Micheli K, Komninos I, Bagkeris E, et al. Sleep patterns in late pregnancy and risk of preterm birth and fetal growth restriction. Epidemiology. 2011;22(5):738-44.

44. Howe LD, Signal TL, Paine SJ, et al. Self-reported sleep in late pregnancy in relation to birth size and fetal distress: the $\mathrm{E}$ Moe, Mama prospective cohort study. BMJ Open. 2015;5(10), e008910.

45. Olivarez SA, Ferres M, Antony K, et al. Obstructive sleep apnea screening in pregnancy, perinatal outcomes, and impact of maternal obesity. Am J Perinatol. 2011;28(8):651-8.

46. Fung AM, Wilson DL, Lappas M, et al. Effects of maternal obstructive sleep apnoea on fetal growth: a prospective cohort study. PLoS One. 2013;8(7), e68057.
47. Bassan H, Uliel-Sibony S, Katsav S, et al. Maternal sleep disordered breathing and neonatal outcome. Isr Med Assoc J. 2016;18(1):45-8.

48. Facco FL, Ouyang DW, Zee PC, et al. Implications of sleepdisordered breathing in pregnancy. Am J Obstet Gynecol. 2014;210(6):559. e1-6.

49. Pamidi S, Marc I, Simoneau G, et al. Maternal sleep-disordered breathing and the risk of delivering small for gestational age infants: a prospective cohort study. Thorax. 2016;71(8):719-25.

50. Ko HS, Kim MY, Kim YH, et al. Obstructive sleep apnea screening and perinatal outcomes in Korean pregnant women. Arch Gynecol Obstet. 2013;287(3):429-33.

51. Koken G, Sahin FK, Cosar E, et al. Oxidative stress markers in pregnant women who snore and fetal outcome: a case control study. Acta Obstet Gynecol Scand. 2007;86(11):1317-21.

52. Na-Rungsri K, Lertmaharit S, Lohsoonthorn V, et al. Obstructive sleep apnea and the risk of preterm delivery. Sleep Breath. 2016.

53. Lee KA, Gay CL. Sleep in late pregnancy predicts length of labor and type of delivery. Am J Obstet Gynecol. 2004;191(6):2041-6.

54. Wangel AM, Molin J, Ostman M, et al. Emergency cesarean sections can be predicted by markers for stress, worry and sleep disturbances in first-time mothers. Acta Obstet Gynecol Scand. 2011;90(3):238-44.

55. Ko YL, Lin PC, Chen SC. Stress, sleep quality and unplanned Caesarean section in pregnant women. Int $\mathrm{J}$ Nurs Pract. 2015;21(5):454-61.

56. Chien LY, Ko YL. Fatigue during pregnancy predicts caesarean deliveries. J Adv Nurs. 2004;45(5):487-94.

57. Powe CE, Levine RJ, Karumanchi SA. Preeclampsia, a disease of the maternal endothelium: the role of antiangiogenic factors and implications for later cardiovascular disease. Circulation. 2011;123(24):2856-69.

58. Izci-Balserak B, Pien GW. Sleep-disordered breathing and pregnancy: potential mechanisms and evidence for maternal and fetal morbidity. Curr Opin Pulm Med. 2010;16(6):574-82.

59. Sibai B, Dekker G, Kupferminc M. Pre-eclampsia. Lancet. 2005;365(9461):785-99.

60. Okun ML, Roberts JM, Marsland AL, et al. How disturbed sleep may be a risk factor for adverse pregnancy outcomes. Obstet Gynecol Surv. 2009;64(4):273-80.

61. Ravishankar S, Bourjeily G, Lambert-Messerlian G, et al. Evidence of placental hypoxia in maternal sleep disordered breathing. Pediatr Dev Pathol. 2015;18(5):380-6.

62. Bourjeily G, Curran P, Butterfield K, et al. Placenta-secreted circulating markers in pregnant women with obstructive sleep apnea. J Perinat Med. 2015;43(1):81-7.

63. Edwards N, Blyton DM, Kirjavainen T, et al. Nasal continuous positive airway pressure reduces sleep-induced blood pressure increments in preeclampsia. Am J Respir Crit Care Med. 2000;162(1):252-7.

64. Poyares D, Guilleminault C, Hachul $\mathrm{H}$, et al. Pre-eclampsia and nasal CPAP: part 2. Hypertension during pregnancy, chronic snoring, and early nasal CPAP intervention. Sleep Med. 2007;9(1):1521.

65. Guilleminault C, Palombini L, Poyares D, et al. Pre-eclampsia and nasal CPAP: part 1. Early intervention with nasal CPAP in pregnant women with risk-factors for pre-eclampsia: preliminary findings. Sleep Med. 2007;9(1):9-14.

66. Morgan BJ. Acute and chronic cardiovascular responses to sleep disordered breathing. Sleep. 1996;19:S206-9.

67. Edwards N, Blyton DM, Kirjavainen TT, et al. Hemodynamic responses to obstructive respiratory events during sleep are augmented in women with preeclampsia. Am J Hypertens. 2001;14(11 Pt 1): $1090-5$. 
68. Blyton DM, Sullivan CE, Edwards N. Reduced nocturnal cardiac output associated with preeclampsia is minimized with the use of nocturnal nasal CPAP. Sleep. 2004;27(1):79-84.

69. Reid J, Taylor-Gjevre R, Gjevre J, et al. Can gestational hypertension be modified by treating nocturnal airflow limitation? J Clin Sleep Med. 2013;9(4):311-7.

70. Whitehead C, Tong S, Wilson D, et al. Treatment of early-onset preeclampsia with continuous positive airway pressure. Obstet Gynecol. 2015;125(5):1106-9.
71. Blyton DM, Skilton MR, Edwards N, et al. Treatment of sleep disordered breathing reverses low fetal activity levels in preeclampsia. Sleep. 2013;36(1):15-21.

72. Facco FL, Parker CB, Reddy UM, et al. NuMoM2b sleepdisordered breathing study: objectives and methods. Am J Obstet Gynecol 2015;212(4):542 e1-127. This study describes the methods of the first multicenter trial investigating SDB and pregnancy outcomes. 